\title{
Enhancing the efficiency of Bortezomib conjugated to pegylated gold nanoparticles: an in vitro study on human pancreatic cancer cells and adenocarcinoma human lung alveolar basal epithelial cells
}

Sílvia Castro Coelho ${ }^{1, *}$, Gabriela M. Almeida ${ }^{2,3}$, Filipe Santos-Silva ${ }^{2,4,5,6}$, Maria Carmo Pereira ${ }^{1}$, Manuel A.N. Coelho ${ }^{1}$

(1) LEPABE, Department of Chemical Engineering, Faculty of Engineering, University of Porto, Portugal

(2) Instituto de Investigação e Inovação em Saúde, Universidade do Porto, Portugal

(3) Expression Regulation in Cancer Group, IPATIMUP, Porto, Portugal

(4) Public Awareness of Cancer Unit, IPATIMUP, Porto, Portugal

(5) Faculty of Medicine, University of Porto, Porto, Portugal,

(6) Department of Biochemistry and Molecular Biology, Eppley Institute, University of Nebraska Medical Center, Omaha, USA

* corresponding author: Sílvia Castro Coelho, LEPABE, Department of Chemical Engineering, Faculty of Engineering, University of Porto, Rua Roberto Frias, PT-4200-465 Porto, Portugal. Phone: +351 225081679; Fax: +351 225081449; E-mail: silvia.coelho@fe.up.pt

Objectives: Gold nanoparticles have become promising vectors for cancer diagnosis and treatment. The present study investigates the effect of Bortezomib (BTZ), a proteasome inhibitor, conjugated with pegylated gold nanoparticles (PEGAuNPs) in pancreatic and lung cancer cells.

Methods: Synthesized gold nanoparticles (PEGAuNPs) were conjugated with bortezomib antitumor drug. We investigated the cytotoxicity induced by Bortezomib conjugated with functionalized gold nanoparticles in vitro, in the human pancreatic (S2-013) and lung (A549) cancer cell lines.

Results: We found an efficient of conjugation of BTZ with PEGAuNPs. In vitro assays showed that after 72 hours' incubation with PEGAuNPs-BTZ cancer cells revealed alterations in morphology; also for S2-013 and A549 cancer cells, the $\mathrm{IC}_{50}$ value of free $\mathrm{BTZ}$ is respectively 1.5 and 4.3 times higher than the $\mathrm{IC}_{50}$ value of PEGAuNPs-BTZ. Furthermore, for TERT-HPNE, the $\mathrm{IC}_{50}$ value is around 63 times lower for free $\mathrm{BTZ}$ than to the conjugated nanovehicle. Cell growth inhibition results showed a remarkable enhancement in the effect of BTZ when conjugated with AuNPs.

Conclusions: Our findings showed that conjugation with PEGAuNPs enhance the BTZ growth-inhibition effect on human cancer cells (S2-013 and A549) and decreases its toxicity against normal cells (TERT-HPNE).

Keywords: bortezomib, gold nanoparticles, pancreatic cancer, lung cancer 


\section{Introduction}

The research on nanotechnology-based delivery carriers opened new promising strategies for targeted drug delivery and diagnosis of various tumours [1]. Recently, the innovation on drug nanocarriers was focused on the challenging limitations associated with multidrug resistance (MDR) and dose-toxicity [2]. The undesired effects of conventional chemotherapy and radiotherapy associated with unfavorable drug pharmacokinetics and pharmacodynamics fostered the design and testing of novel delivery systems for a myriad of antitumor drugs [3-5]. Compared to chemotherapeutic agents, nanocarriers can improve tumour specificity and intensify drug efficacy [4]. Nanovehicles can passively target solid tumours due to the enhanced permeability and retention (EPR) effect in these neoplastic tissues [2, 3]. They are able to be internalized in different cell cycle phases [6]. Amongst these drug delivery systems (DDS), gold nanoparticles are a novel and promising carrier due to the enhanced stability and reduced toxicity [7-9].

Colloidal gold nanoparticles (AuNPs) have been extensively studied for different therapeutic applications [3, $10,11]$. They have been distinguished because of their optical properties as a high surface area to volume ratio exhibiting high selectivity and chemical reactivity [12-14]. They present high biocompatibility, stability and easy conjugation, non-toxicity and good uptake on human cells $[15,16]$. Recently, gold-based nanoparticles have been used to develop new agents as cancer imaging probes [17, 18], as agents to enhance radiation therapy [19-22] and as drug delivery systems for cancer treatment [23-25]. Several studies reported that AuNPs in combination with chemotherapy and radiotherapy improve therapy efficacy in different cancer types, including pancreatic and human lung cancers [15, 26-28].

Gold nanoparticles are reported to increase the drug toxicity compared to free drug $[4,15,23,29,30]$. This fact occurs by passive targeting through EPR effect [31]. Pegylation of AuNPs (PEGAuNPs) has been shown to increase the circulation time by reducing the protein absorption and reticuloendothelial system uptake opsonisation, mechanisms associated to low bioavailability [22, 32-34].

Bortezomib (BTZ), a reversible inhibitor of the $26 \mathrm{~S}$ proteasome, indicated to treat patients with multiple myeloma, is known for its antiproliferative, proapoptotic, antiangiogenic and antitumour activities [35]. It inhibits the regulation of basic cellular functions as cell-division cycle, proliferation, differentiation and apoptosis [36]. It is a boronic acid dipeptide reported in preclinical studies with antitumor activity in xenograft models of multiple myeloma, adult T-cell leukemia, cancer of lung, breast, prostate, pancreas, head and neck, colon and melanoma $[22,37,38]$. Nawrocki et al. reported that BTZ sensitizes pancreatic cancer cells 
enhancing the anticancer activity of cisplatin [38]. More recently a phase II preclinical studies indicated a synergy of the BTZ combined with gemcitabine/carboplatin promising for advanced non-small lung cancer [39].

Herein, the role and effect of the conjugation of BTZ with PEGAuNPs for drug delivery on cancer cells were investigated in human pancreatic (S2-013) and lung cancer cells (A549). The release efficiencies were analyzed. The cellular internalization of nanoparticles was visualized and the cellular morphology was assessed. The effect of the conjugation of BTZ with PEGAuNPs on cell growth was assessed on S2-013, A549 and TERT-HPNE cell lines.

2.0 Materials and methods

\subsection{Chemicals}

Bortezomib (VELCADE) was purchased from Selleck Chemicals LLC (USA); fetal bovine serum (FBS), phosphate buffered saline (PBS), trypsin, Dulbecco's modified Eagle's medium (DMEM) was obtained from Invitrogen Co. (Scotland, UK). Acetic acid, dimethyl sulfoxide (DMSO), sulforhodamine B (SRB), trypan blue, trisodium citrate dihydrate and tetrachloroauric (III) acid ( $\mathrm{HAuCl}_{4} ; 99.99 \%$ trace metals basis, $30 \mathrm{wt} \%$ in dilute $\mathrm{HCl}$ ) were purchased from Sigma-Aldrich (Germany). Tricloroacetic acid (TCA) and Tris buffer were acquired from Merck (Darmstadt, Germany). a-thiol-w-carboxyl (polyethylene glycol) (HS-PEG-COOH; molecular weight $394.57 \mathrm{Da}$ ) was purchased from Prochimia (Poland).

\subsection{Synthesis of pegylated gold nanoparticles}

AuNPs were synthesized by the reduction of tetrachloroauric acid $\left(\mathrm{HAuCl}_{4}\right)$ with trisodium citrate - Turkevitch method $[40,41]$. $\mathrm{HAuCl}_{4}$ aqueous solution was heated to slight boiling and trisodium citrate was added for 15 min. Then, AuNPs were pegylated (PEGAuNPs) with thiolated poly(ethylene glycol) carboxylic acid (PEG) layer, with a molar ratio $1: 1,000$, respectively. The resultant solution was centrifuged at $12,000 \times \mathrm{g}$ during 10 min to remove the unbound PEG molecules. The concentration of the PEGAuNPs, determined by the LambertBeer Law assuming the molar absorptivity of the AuNPs plasmon resonance band at $526 \mathrm{~nm}$ being $2.33 \times 10^{8} \mathrm{M}^{-}$ ${ }^{1} \mathrm{~cm}^{-1}$, was $15.08 \mathrm{nM}$.

\subsection{Conjugation of bortezomib to PEGAuNPs, PEGAuNPs-BTZ}

For the conjugation of bortezomib (BTZ) to pegylated gold nanoparticles, the EDC/NHSS-mediated amine coupling reaction was followed [28]. The molar ratio PEGAuNPs:Bortezomib is 1:1540. The solution was 
stirred at room temperature for $1 \mathrm{~h}$. The final solution was centrifuged at 12,000 $\mathrm{x}$ g during $10 \mathrm{~min}$ to remove the unbound antitumour drug.

\subsection{Dynamic light scattering and electrophoretic mobility measurements}

Size distribution and zeta potential of the nanoparticles was analyzed by dynamic light scattering (DLS) and laser Doppler velocimetry, respectively, by a Zetasizer Nano ZS (Malvern Instruments Ltd., Malvern, UK), at $25^{\circ} \mathrm{C}$. Size measurements were performed at a scattering angle of 173 in a $12 \mathrm{~mm}$ square polystyrene cuvette (Sarstedt, Germany). The zeta potential was obtained by using a disposable capillary cell (DTS 1060, Malvern).

\subsection{UV-VIS spectroscopy}

The UV-VIS absorption spectra of the PEGAuNPs and PEGAuNPs-BTZ were measured in a $1 \mathrm{~cm}$ quartz cuvette at room temperature using a Shimadzu UV-1700 PharmaSpec spectrophotometer.

\subsection{Attenuated Total Reflectance-Fourier Transform Infrared Spectroscopy (ATR-FTIR)}

The PEGAuNPs-BTZ, PEGAuNPs and BTZ solutions were analysed and ATR-FTIR spectra were recorded with an ALPHA FTIR Spectrometer (Bruker) in the spectral range 4,000-400 $\mathrm{cm}^{-1}$, resolution of $4 \mathrm{~cm}^{-1}$ and 64 scans, at room temperature.

\subsection{Assay of 20S Proteasome Activity}

BTZ activity was studied with 20S proteasome as described in the 20S proteasome assay kit-BML-AK740 (Enzo Life Sciences). It was performed a fluorescence standard curve of BTZ for concentrations between 20 $\mathrm{nM}$ and $2.5 \mu \mathrm{M}$.

Incubation assays with supernatants of PEGAuNPs-BTZ were performed at $37^{\circ} \mathrm{C}$, during $50 \mathrm{~min}$. BTZ activity was monitored as the reaction substrate's fluorescence emission (excitation at $360 \mathrm{~nm}$, emission at $485 \mathrm{~nm}$ ) using a microplate reader (PowerWave HT Microplate Spectrophotometer, BioTek), in order to determine the encapsulation efficiency of BTZ.

\subsection{In vitro drug release studies}

The drug release study of PEGAuNPs-BTZ was carried out at $37^{\circ} \mathrm{C}$ by dialysis using a regenerated cellulose membrane with an initial BTZ concentration of $17 \mu \mathrm{M}$, respectively. The solutions were incubated in $6 \mathrm{~mL}$ of PBS 0.01 M, with constant magnetic stirring. The BTZ concentration of the dialysate buffer was analyzed with time using spectrophotometric analysis at $270 \mathrm{~nm}$ (the concentration is under the limit of detection of the Shimadzu UV-1700 PharmaSpec spectrophotometer).

\subsection{Cell lines}


The pancreatic cell lines S2-013 (well differentiated tubular adenocarcinoma and moderately metastatic subline cloned from the human pancreatic tumor cell line SUIT-2) and hTERT-HPNE (immortalized human pancreatic duct epithelial cell line) were kindly provided by Prof. M. A. Hollingsworth (Eppley Institute for Research in Cancer and Allied Diseases, UNMC, Omaha, NE, USA). Adherent cell cultures were maintained in DMEM with $10 \%$ heat-inactivated $\mathrm{FBS}$ at $37^{\circ} \mathrm{C}$ in a humidified atmosphere of $5 \% \mathrm{CO}_{2}$ incubator.

The adenocarcinoma human lung cancer cell line A549 was kindly provided by Dr George DD Jones (University of Leicester, UK). A549 cells were grown as a monolayer in RPMI 1640 media with 10\% heatinactivated $\mathrm{FBS}$ at $37^{\circ} \mathrm{C}$ in a humidified atmosphere of $5 \% \mathrm{CO}_{2}$.

\subsection{Cellular imaging studies}

Imaging experiments were performed with the S2-013 and A549 cells. About 800 cells per well were grown for $24 \mathrm{~h}$ in $\mu$-chamber 12-well plates (ibidi, Germany) under normal conditions $\left(5 \% \mathrm{CO}_{2}\right.$ humidified atmosphere at $37^{\circ} \mathrm{C}$ ). The cells were incubated with PEGAuNPs-BTZ with $0.195 \mu \mathrm{M}$ of BTZ concentration and $0.195 \mu \mathrm{M}$ of BTZ alone for $72 \mathrm{~h}$. Following the incubation, the cells were washed with PBS $0.01 \mathrm{M}$. After, cells were incubated with $200 \mu \mathrm{L}$ of $60 \mathrm{mM}$ lysotracker deep red for $60 \mathrm{~min}$ to stain lysosomes. Then cells were incubated with $200 \mu \mathrm{L}$ of cell mask plasma membrane for $5 \mathrm{~min}$. After washing the cells, they were rinsed with $1 \%$ of phosphate buffered saline (PBS) and fixed using 4\% paraformaldehyde for $15 \mathrm{~min}$. The cells were washed with PBS and mounted in a glycerol-based nondrying and non hardening medium. Imaging was performed using a laser scanning confocal microscope (LSCM) TCS SP5 II (Leica Microsystems, Germany).

To evaluate the influence of nanoparticle surface on the morphology in the cells, the control untreated cells were also imaged. Different areas were analyzed and at least three images were acquired for each type of cell.

\subsection{Evaluation of in vitro cytotoxicity}

The concentrations of PEGAuNPs-BTZ, PEGAuNPs and BTZ that inhibit cell survival in $50 \%\left(\mathrm{IC}_{50}\right)$ and cell growth in $50 \%\left(\mathrm{GI}_{50}\right)$ were determined using the SRB assay, as previously [15]. Briefly, the S2-013, hTERTHPNE and A549 cells were seeded in 96-well plates (1,000 cells per well) under normal conditions $\left(5 \% \mathrm{CO}_{2}\right.$ humidified atmosphere at $37^{\circ} \mathrm{C}$ ) and allowed to adhere for 20-24 h. The cells were then treated for $72 \mathrm{~h}$ with BTZ, PEGAuNPs or PEGAuNPs-BTZ at concentrations ranging between 0.001 and $2.920 \mu \mathrm{M}$ BTZ. Following this incubation period, the cells were fixed with $10 \%$ TCA for $1 \mathrm{~h}$ on ice, washed and stained with $0.4 \%$ SRB dye for $30 \mathrm{~min}$. The cells were then washed repeatedly with $1 \%$ acetic acid to remove unbound dye. The 
protein-bound stain was solubilized with $10 \mathrm{mM}$ Tris solution. The SRB absorbance was measured at $560 \mathrm{~nm}$ using the PowerWave microplate reader and $\mathrm{IC}_{50}$ and $\mathrm{GI}_{50}$ values determined.

\subsection{Statistical analysis}

At least three independent experiments were performed for each assay. Statistical significance $(p<0.05)$ was determined by the Student's t-Test.

\subsection{Results and Discussion}

SEM (Figure 1) presents pegylated gold nanoparticles (PEGAuNPs) spherical in shape and with a small hydrodynamic diameter.

These results are in agreement with the size distribution (PdI 0.4) indicated the synthesis of uniform $39 \pm 1 \mathrm{~nm}$ nanoparticles (NPs). The zeta potential was approximately $-39 \mathrm{mV}$ (Table 1).

The PEGAuNPs-BTZ conjugates were then synthesized through the EDC/NHSS crosslinking of carboxylic acids from PEGAuNPs with secondary amine group of BTZ. To confirm the chemical composition of conjugated nanoparticles, ATR-FTIR spectra of BTZ loading on PEGAuNPs were obtained (Figure 2A). On Figure 2A, the ATR-FTIR spectrum of unmodified PEGAuNPs showed characteristic peaks at $1750 \mathrm{~cm}^{-1}$ from carbonyl $\mathrm{C}=\mathrm{O}$ stretching and at $1077 \mathrm{~cm}^{-1}$ from $\mathrm{C}-\mathrm{O}$ stretching of the ethylene glycol monomers. In the spectrum of PEGAuNPs-BTZ, the peak at $1635 \mathrm{~cm}^{-1}$ indicates the $\mathrm{C}=\mathrm{N}$ that can be assigned to the imine vibration (Figure 2A). The peaks at $1443 \mathrm{~cm}^{-1}$ and $1492 \mathrm{~cm}^{-1}$ indicate the $\mathrm{C}=\mathrm{C}$ stretch from aromatic group and the N-H bending from secondary amide, respectively. The peaks at 613 and $3262 \mathrm{~cm}^{-1}$ reflect the B-O-H stretching of boronic acid, belonging to the BTZ. At $770 \mathrm{~cm}^{-1}$ it is visible the peaks of C-H aromatic out-ofplane bending. The presence of a surface plasmon resonance band at ca. $526 \mathrm{~nm}$ conforms the formation of spherical gold nanoparticles and the BTZ characteristic band was observed at $270 \mathrm{~nm}$. (data not shown). Bortezomib conjugation efficiency was investigated with $20 \mathrm{~S}$ proteasome assay measuring the proteasome activity. According to the results, $92.0 \pm 0.7 \%(\mathrm{w} / \mathrm{w})$ of BTZ was conjugated with PEGAuNPs. The final BTZ concentration in solution is $18.0 \pm 0.2 \mu \mathrm{M}$.

The stability of PEGAuNPs-BTZ for 72 hours was evaluated in phosphate buffer solution (pH 7.4) at $37^{\circ} \mathrm{C}$, which simulates the physiological conditions (Figure 3).

It is observed that BTZ has an initial delay over the first hours. After this, Figure 3 indicates the slow and controlled release of the drug. The conjugated PEGAuNPs release about $30 \%$ of the BTZ for 72 hours. Also, 
for the same period of time, all amount of BTZ free without PEGAuNP presence was released. The PEGAuNPs exhibit a high controlled release of BTZ, with the release rate measured to be around 3 times lower than that of free BTZ.

To investigate the morphology of cancer cells - S2-013 and A549 cells, cancer cells were incubated for 72 hours in the presence of PEGAuNPs-BTZ. Confocal microscopy images confirm that the internalization to the cytoplasm corresponded to significant modifications on cells appearance (size and morphology) (Figure 4). The nuclei of the cells are visualized with DAPI staining. It was observed the intracellular accumulation of nanocarriers, clearly encompassing the acidified membrane compartments in cytoplasm of S2-013 and A549 (Figure 4B and Figure 4E). Comparing Figure 4B and Figure 4E to Figure 4C and Figure 4F, respectively, it is visible irregular cellular shape in comparison to control cells, which might indicating the more efficient apoptotic effect of the conjugated NPs than with free BTZ. The enrichment of the uptaken PEGAuNPs-BTZ in the lysosomal pathway was considered. Apoptotic characteristics such as rounding, shrinkage were observed revealing typical apoptotic process. This fact corroborated with the higher increase in the in vitro cytotoxicity verified to cells incubated with conjugated BTZ than with free BTZ (Figure 5).

The cytotoxicity effect of PEGAuNPs-BTZ and free BTZ was studied against S2-013 cells, TERT-HPNE cells and A549 cells. It was possible to compare the cytotoxicity response following exposure to different concentrations of PEGAuNPs-BTZ on different type of cells. Cell survival was evaluated with the SRB test to compare the toxicity of PEGAuNPs-BTZ. Figures 5 shows the cell survival results of the cell lines after incubation with functionalized PEGAuNPs and free BTZ for 72 hours with increasing concentration. Previous studies have demonstrated that PEGAuNPs did not affect the cell survival for the concentration range of 0.1 to $1.0 \mathrm{nM}[15,23]$. The cell survival of S2-013 and TERT-HPNE cells after exposure was found to be in the range of $42-5 \%$ and $90-38 \%$, respectively, for a range of BTZ concentration of 0.01-2.93 $\mu \mathrm{M}$ (Figure 5). With the same cell lines incubated with equal amount of free BTZ, it showed cell survival in the range of 57$0.8 \%$ and 84-2.6\%, respectively. On S2-013, at each concentration, in particular, for BTZ concentration between 0.01 and $0.1 \mu \mathrm{M}$, cytotoxicity of conjugated BTZ was significantly higher than that of free BTZ. This increase in the cytotoxicity of conjugated BTZ with PEGAuNPs compared with free BTZ might occurred probably due to the enhanced cellular uptake via endocytic process combined with high accumulation of the nanosystem in the acidic organelles of the cancer cells. This result did not occur for normal pancreatic cell lines as it was shown in Figure 5B. In fact, at these concentrations, PEGAuNPs-BTZ are much less toxic in the 
percentage of cell growth for the HPNE cells than free BTZ. Also, it was found that cell survival decreased by $38-12 \%$ after assembling the PEGAuNPs-BTZ that those that used only free drugs (75-48 \% of cell growth, respectively) at the concentrations of $0.01-0.05 \mu \mathrm{M}$ for A549s (Figure 5). The determined $\mathrm{IC}_{50}$ values for S2013, TERT-HPNE and A549 cells were $0.0082 \pm 0.0002 \mu \mathrm{M}, 0.4299 \pm 0.0384 \mu \mathrm{M}$ and $0.0066 \pm 0.0005 \mu \mathrm{M}$, respectively by PEGAuNPs-BTZ. These values are significantly different from free $\mathrm{BTZ} \mathrm{IC}_{50}$ (Table 2), which could be explained by the increasing of the drug efficiency on suspension on S2-013 and A549.

On TERT-HPNE, the opposite effect is verified which might be due to protection of drug by PEGAuNPs. The BTZ concentrations inhibiting cell growth in $50 \%$ (GI 50 values) were around $0.0023 \pm 0.0007 \mu \mathrm{M}$ and 0.0054 $\pm 0.0006 \mu \mathrm{M}$ for S2-013 and A549, respectively (Table 2). For TERT-HPNEs, GI $5_{00}$ value is $0.0535 \pm 0.0020$ $\mu \mathrm{M}$. These results suggest that PEGAuNPs-BTZ show higher toxicity effect on cancer cells in comparison with normal cells. The effectiveness of PEGAuNPs-BTZ in targeting cancer cells was observed on cell growth of S2-013 and A549 (Table 2).

\subsection{Conclusions}

In summary, this work reports the preparation of bortezomib-conjugated gold nanoparticles for cancer therapy. The results suggested that PEGAuNPs-BTZ has a better potential than free BTZ on cancer cell growthinhibition. More important, our present data indicated that it is possible to reduce the PEGAuNPs-BTZ concentration 4.3 and 1.5 times to have the same effect as that of the free drug (BTZ) for A549 and S2-013 cells, respectively. By other hand in TERT-HPNE cells, it is necessary to increase around 63 times the concentration of PEGAuNPs-BTZ to have the same effect that free drug induces.

To have a better understanding of this novel drug conjugated PEGAuNPs, it is envisaged to test the drug delivery system in other cancer cell lines (colon, prostate, breast) foreseeing in vivo studies.

\subsection{Acknowledgements}

This work was supported by financial support by TRANSCAN-FCT (research project

TRANSCAN/0001/2012) and Portuguese Cancer League. The authors wish to thank Michael A.

Hollingsworth, PhD and the UNMC Pancreatic SPORE (2 P50 CA127297) for providing cell lines (S2-013 and Tert-HPNE) used in some of the experiments described in this article. We gratefully acknowledge Dr George DD Jones (University of Leicester, UK) for kindly providing the human NSCLC cell line A549. We would like 
to thank Dr. Paula Sampaio for the collaboration with confocal work analysis. IPATIMUP integrates the i3S

Research Unit, which is partially supported by FCT, the Portuguese Foundation for Science and Technology.

GM Almeida was supported by the Investigator FCT Program 2013 (IF/00615/2013), POPH - QREN Type 4.2,

European Social Fund and Portuguese Ministry of Science and Technology (MCTES).

\subsection{References}

1. Ali MRK, Panikkanvalappil SR, El-Sayed MA. Enhancing the Efficiency of Gold Nanoparticles Treatment of Cancer by Increasing Their Rate of Endocytosis and Cell Accumulation Using Rifampicin. Journal of the American Chemical Society 2014 2014/03/26;136:4464-67.

2. Dong X, Mumper RJ. Nanomedicinal strategies to treat multidrug-resistant tumors: current progress. Nanomedicine (Lond) 2010 Jun;5:597-615.

3. Coelho SC, Pereira MC, Juzeniene A, et al. Supramolecular nanoscale assemblies for cancer diagnosis and therapy. Journal of controlled release : official journal of the Controlled Release Society 2015 Jul 6;213:15267.

4. Nichols JW, Bae YH. Nanotechnology for Cancer Treatment: Possibilities and Limitations. In: Bae HY, Mrsny JR, Park K, eds. Cancer Targeted Drug Delivery: An Elusive Dream. New York, NY: Springer New York 2013:37-56.

5. Izar B, Rotow J, Gainor J, et al. Pharmacokinetics, clinical indications, and resistance mechanisms in molecular targeted therapies in cancer. Pharmacol Rev 2013;65:1351-95.

6. Kim JA, Aberg C, Salvati A, et al. Role of cell cycle on the cellular uptake and dilution of nanoparticles in a cell population. Nat Nano 2012 01//print; 7:62-68.

7. Patel S, Bhirde AA, Rusling JF, et al. Nano Delivers Big: Designing Molecular Missiles for Cancer Therapeutics. Pharmaceutics 2011;3:34-52.

8. Gelperina S, Kisich K, Iseman MD, et al. The Potential Advantages of Nanoparticle Drug Delivery Systems in Chemotherapy of Tuberculosis. American Journal of Respiratory and Critical Care Medicine 2005;172:1487-90.

9. Tiwari G, Tiwari R, Sriwastawa B, et al. Drug delivery systems: An updated review. International Journal of Pharmaceutical Investigation 2012 Jan-Mar;2:2-11.

10. Ghosh P, Han G, De M, et al. Gold nanoparticles in delivery applications. Advanced Drug Delivery Reviews 2008;60:1307-15.

11. Cai W, Gao T, Hong H, et al. Applications of gold nanoparticles in cancer nanotechnology. Nanotechnology, science and applications 2008 09/;2008:10.2147/NSA.S3788.

12. Li J-L, Wang L, Liu X-Y, et al. In vitro cancer cell imaging and therapy using transferrin-conjugated gold nanoparticles. Cancer Letters 2009 2/18/;274:319-26.

13. Yang F, Jin C, Subedi S, et al. Emerging inorganic nanomaterials for pancreatic cancer diagnosis and treatment. Cancer Treatment Reviews 2012 10//;38:566-79.

14. Su S, Zuo X, Pan D, et al. Design and applications of gold nanoparticle conjugates by exploiting biomolecule-gold nanoparticle interactions. Nanoscale 2013 Apr 7;5:2589-99.

15. Coelho SC, Rocha S, Juzenas P, et al. Gold nanoparticle delivery-enhanced proteasome inhibitor effect in adenocarcinoma cells. Expert opinion on drug delivery 2013;10:1345-52.

16. Zhao J, Lee P, Wallace MJ, et al. Gold Nanoparticles in Cancer Therapy: Efficacy, Biodistribution, and Toxicity. Current pharmaceutical design 2015;21:4240-51.

17. Popovtzer R, Agrawal A, Kotov NA, et al. Targeted Gold Nanoparticles enable Molecular CT Imaging of Cancer. Nano letters 2008;8:4593-96.

18. Huang X, El-Sayed MA. Gold nanoparticles: Optical properties and implementations in cancer diagnosis and photothermal therapy. Journal of Advanced Research 2010 1//;1:13-28.

19. Juzenas P, Chen W, Sun Y-P, et al. Quantum dots and nanoparticles for photodynamic and radiation therapies of cancer. Advanced Drug Delivery Reviews 2008;60:1600-14.

20. Antosh MP, Wijesinghe DD, Shrestha S, et al. Enhancement of radiation effect on cancer cells by goldpHLIP. Proceedings of the National Academy of Sciences 2015 April 28, 2015;112:5372-76. 
21. Mesbahi A. A review on gold nanoparticles radiosensitization effect in radiation therapy of cancer. Reports of Practical Oncology \& Radiotherapy 2010 11//;15:176-80.

22. Coelho SC, Rocha S, Pereira MC, et al. Enhancing proteasome-lnhibitor effect by functionalized gold nanoparticles. Journal of biomedical nanotechnology 2014 Apr;10:717-23.

23. Coelho SC, Almeida GM, Pereira MC, et al. Functionalized gold nanoparticles improve afatinib delivery into cancer cells. Expert opinion on drug delivery 2016 Jan;13:133-41.

24. Du YQ, Yang XX, Li WL, et al. A cancer-targeted drug delivery system developed with gold nanoparticle mediated DNA-doxorubicin conjugates. RSC Advances 2014;4:34830-35.

25. Duncan B, Kim C, Rotello VM. Gold nanoparticle platforms as drug and biomacromolecule delivery systems. Journal of controlled release : official journal of the Controlled Release Society 2010 Nov 20;148:1227.

26. Sobhan MA, Sreenivasan VKA, Withford MJ, et al. Non-specific internalization of laser ablated pure gold nanoparticles in pancreatic tumor cell. Colloids and Surfaces B: Biointerfaces 2012 4/1/;92:190-95.

27. Kao HW, Lin YY, Chen CC, et al. Biological characterization of cetuximab-conjugated gold nanoparticles in a tumor animal model. Nanotechnology $2014 \mathrm{Jul}$ 25;25:295102.

28. Qian Y, Qiu M, Wu Q, et al. Enhanced cytotoxic activity of cetuximab in EGFR-positive lung cancer by conjugating with gold nanoparticles. Scientific Reports 2014 12/15/online;4:7490.

29. Wojcik M, Lewandowski W, Krol M, et al. Enhancing anti-tumor efficacy of Doxorubicin by noncovalent conjugation to gold nanoparticles - in vitro studies on feline fibrosarcoma cell lines. PloS one 2015;10:e124955.

30. Chen Y, Li N, Yang Y, et al. A dual targeting cyclodextrin/gold nanoparticle conjugate as a scaffold for solubilization and delivery of paclitaxel. RSC Advances 2015;5:8938-41.

31. Vigderman L, Zubarev ER. Therapeutic platforms based on gold nanoparticles and their covalent conjugates with drug molecules. Advanced Drug Delivery Reviews 2013 5//;65:663-76.

32. Verma A, Stellacci F. Effect of surface properties on nanoparticle-cell interactions. Small 2010 Jan;6:12-

21.

33. Kumar A, Zhang X, Liang XJ. Gold nanoparticles: emerging paradigm for targeted drug delivery system. Biotechnology advances 2013 Sep-Oct;31:593-606.

34. Dykman LA, Khlebtsov NG. Uptake of Engineered Gold Nanoparticles into Mammalian Cells. Chemical Reviews 2014 2014/01/22;114:1258-88.

35. Boccadoro M, Morgan G, Cavenagh J. Preclinical evaluation of the proteasome inhibitor bortezomib in cancer therapy. Cancer cell international 2005 Jun 1;5:18.

36. Piperdi B, Ling YH, Liebes L, et al. Bortezomib: understanding the mechanism of action. Mol Cancer Ther 2011 Nov;10:2029-30.

37. Hideshima T, Richardson $\mathrm{P}$, Chauhan D, et al. The proteasome inhibitor PS-341 inhibits growth, induces apoptosis, and overcomes drug resistance in human multiple myeloma cells. Cancer Res 2001 Apr 1;61:3071-6.

38. Los M, Gibson SB. Apoptotic Pathways as Targets for Novel Therapies in Cancer and Other Diseases: Springer US, 2006.

39. Davies AM, Chansky K, Lara PN, Jr., et al. Bortezomib plus gemcitabine/carboplatin as first-line treatment of advanced non-small cell lung cancer: a phase II Southwest Oncology Group Study (S0339). Journal of thoracic oncology : official publication of the International Association for the Study of Lung Cancer 2009 Jan;4:87-92.

40. Turkevich J, Stevenson PC, Hillier J. A study of the nucleation and growth processes in the synthesis of colloidal gold. Discussions of the Faraday Society 1951;11:55-75.

41. Kimling J, Maier M, Okenve B, et al. Turkevich Method for Gold Nanoparticle Synthesis Revisited. The Journal of Physical Chemistry B 2006;110:15700-07. 


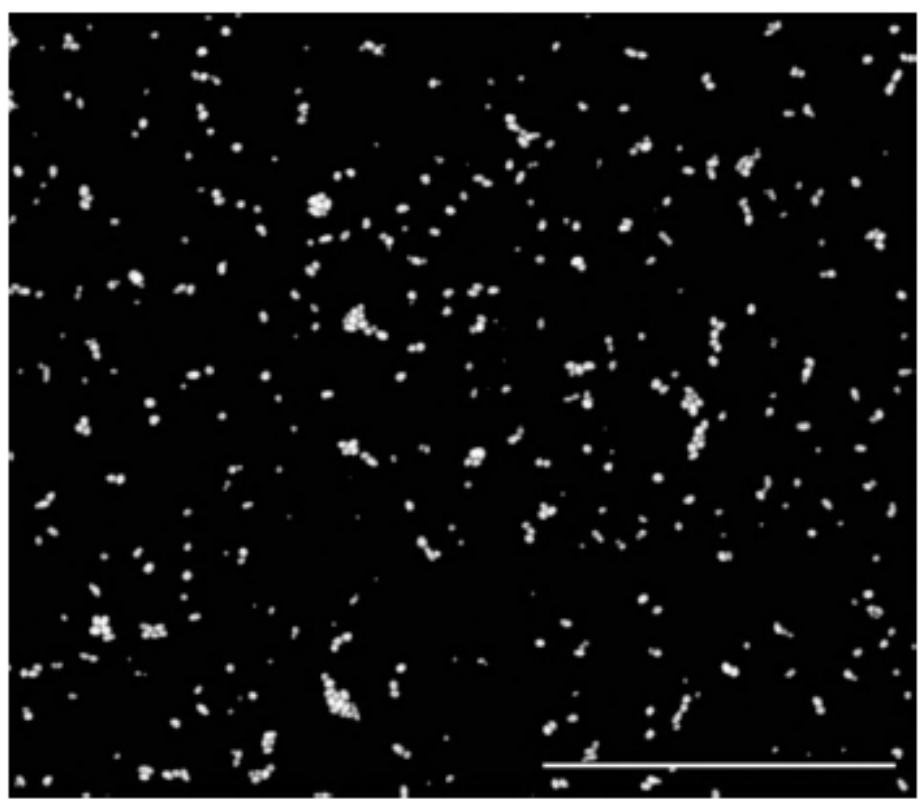

Figure 1 PEGAuNPs SEM image. Scale bar is $1 \mu \mathrm{m}$.

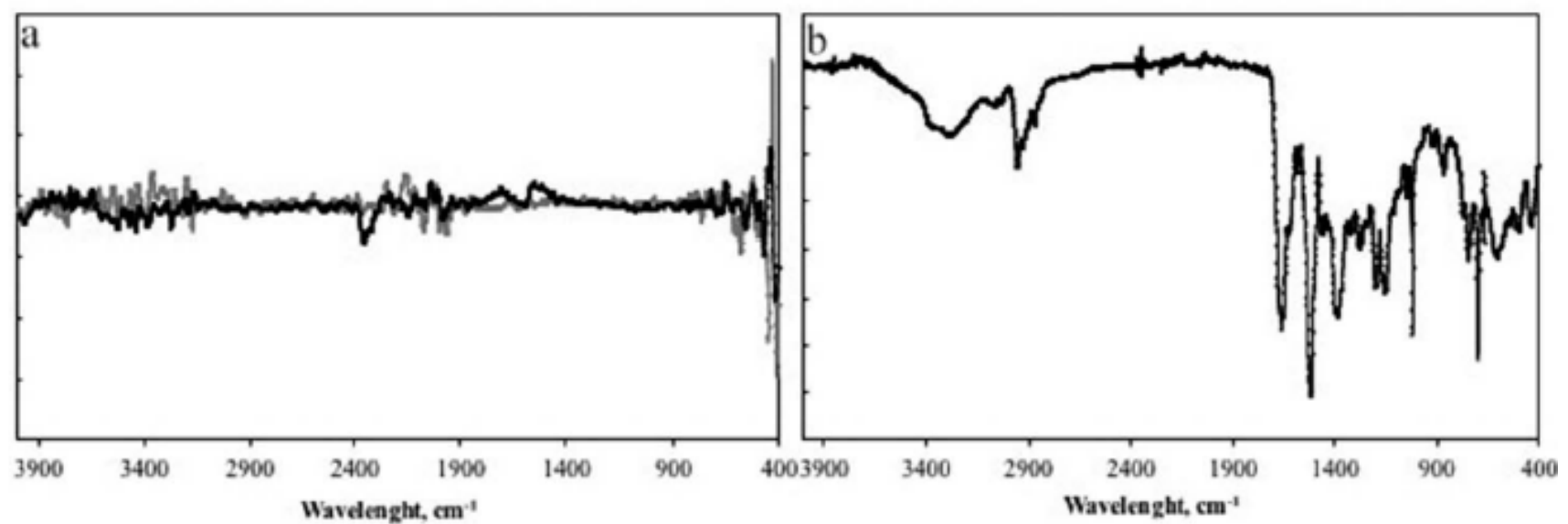

Figure 2 A) FTIR spectra of PEGAuNPs (black line) and PEGAuNPs-BTZ (grey line). B) FTIR spectra of Bortezomib. The spectra were shifted for a better visualization.

PEGAuNPs: Pegylated gold nanoparticles

PEGAuNPs-BTZ: Conjugated pegylated gold nanoparticles with Bortezomib 


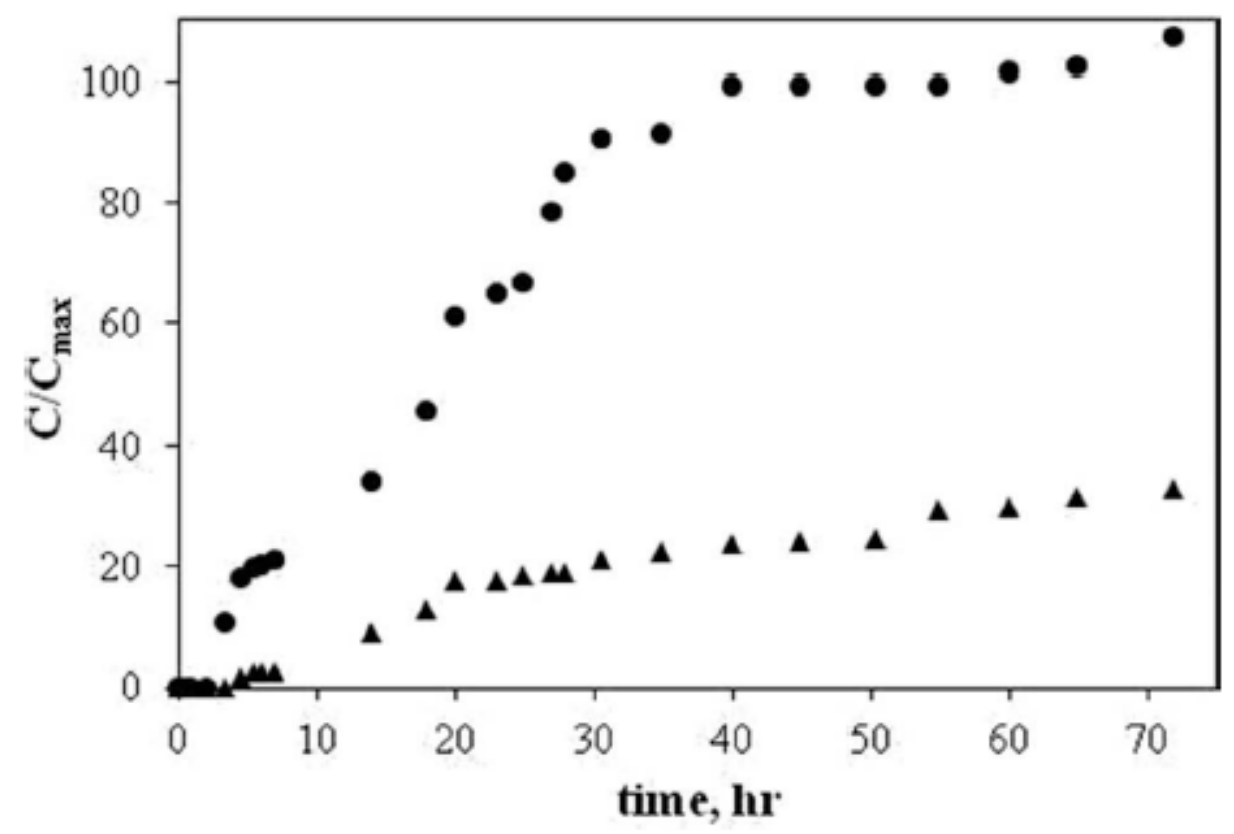

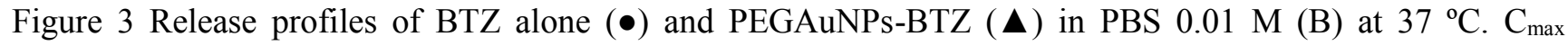
corresponds to the total amount of BTZ added.

BTZ: Bortezomib

PEGAuNPs-BTZ: Conjugated pegylated gold nanoparticles with Bortezomib
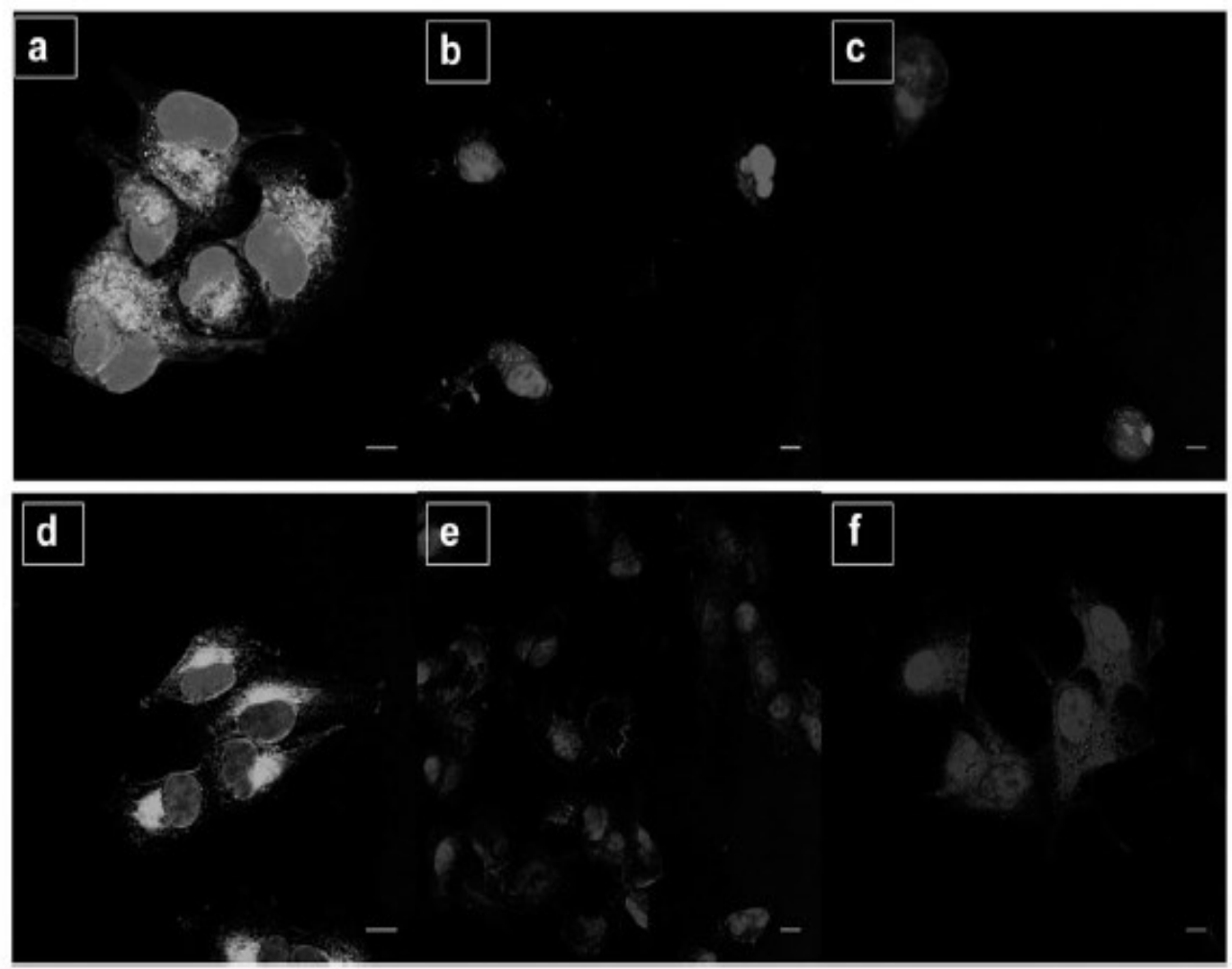

Figure 4 Confocal images of the S2-013 (A, B, C) and A549 (D, E, F) cells after $72 \mathrm{~h}$ incubation. (A, D) the control untreated cells; (B, E) the cells were incubated with PEGAuNPs-BTZ with $0.195 \mu \mathrm{M}$ and (C, F) the cells were incubated with $0.195 \mu \mathrm{M}$ BTZ alone. Scale bar in all images is $10 \mu \mathrm{M}$.

PEGAuNPs-BTZ: Conjugated pegylated gold nanoparticles with Bortezomib BTZ: Bortezomib 


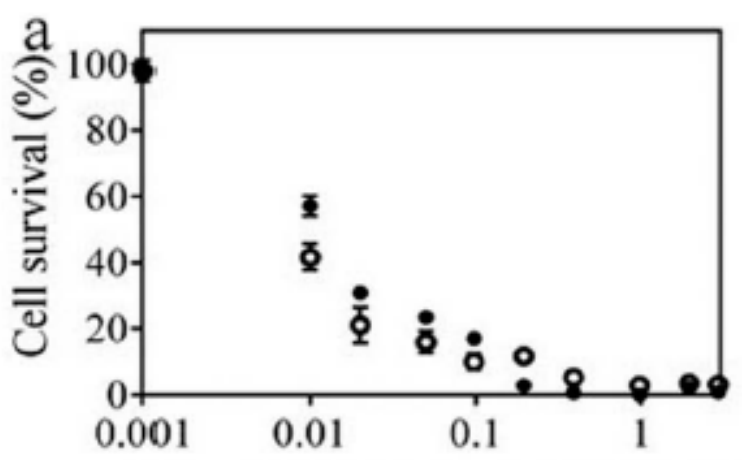

$\log ($ Concentration of BTZ) $(\mu \mathrm{M})$

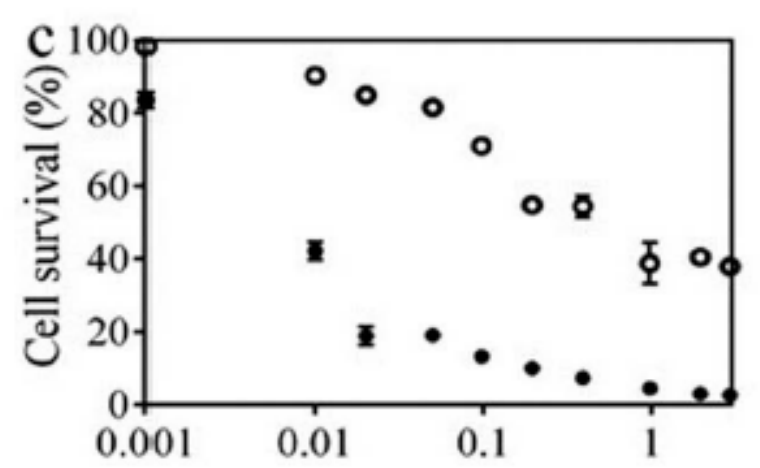

$\log ($ Concentration of BTZ) $(\mu \mathrm{M})$

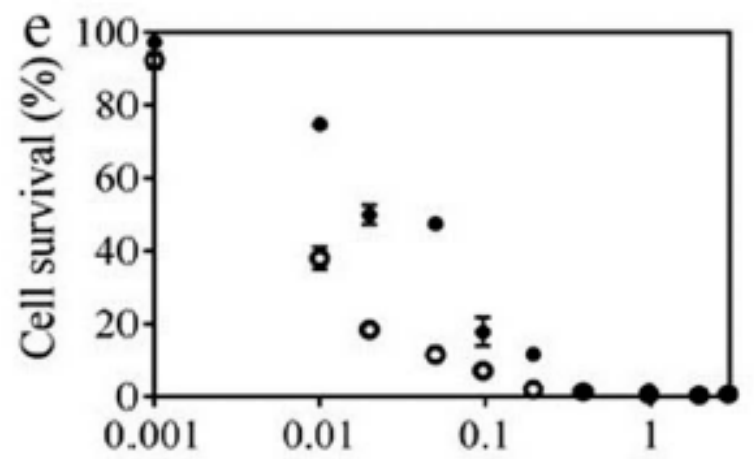

Log (Concentration of BTZ) $(\mu \mathrm{M})$

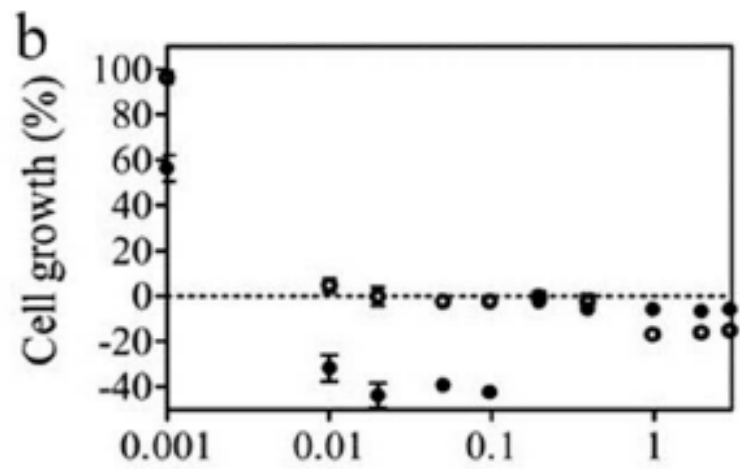

$\log$ (Concentration of BTZ) $(\mu \mathrm{M})$

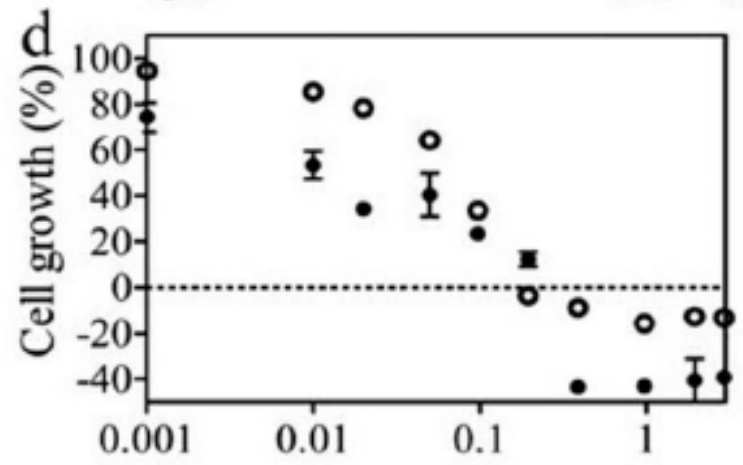

$\log ($ Concentration of BTZ) $(\mu \mathrm{M})$

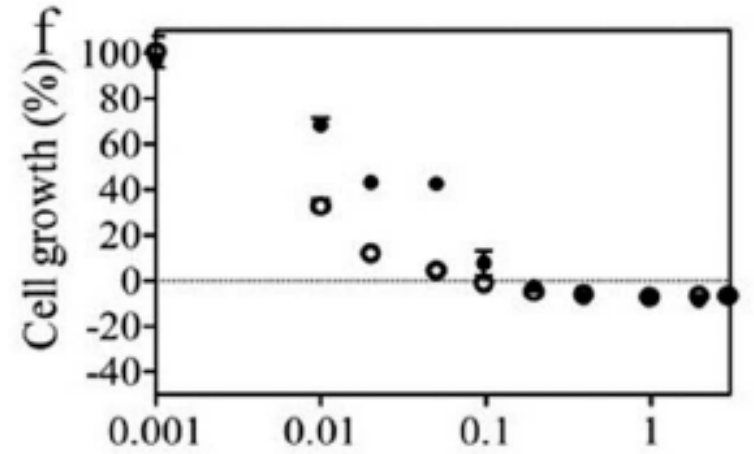

$\log ($ Concentration of BTZ) $(\mu \mathrm{M})$

Figure 5 Effect of the PEGAuNPs-BTZ $(\circ)$ and BTZ alone $(\bullet)$ on the cell survival ( $\mathrm{IC}_{50}$ determination) (A, C, E) and on cell growth ( $\mathrm{GI}_{50}$ determination) $(\mathrm{B}, \mathrm{D}, \mathrm{F})$ of S2-013 (A, B), hTERT-HPNE (C, D) and A-549 (E, F) cells.

PEGAuNPs-BTZ: Conjugated pegylated gold nanoparticles with Bortezomib BTZ: Bortezomib 
Table 1 Hydrodynamic diameter, polydispersity index (PdI) and zeta potential of gold nanoparticles.

PEGAuNPs: Pegylated gold nanoparticles

PEGAuNPs-BTZ: Conjugated pegylated gold nanoparticles with Bortezomib

\begin{tabular}{|c|c|c|c|c|c|}
\hline Sample & Hydrodynamic diameter, $\mathrm{nm}$ & Pdl & Zeta potential, $\mathrm{mV}$ & AuNPs concentration, $\mathrm{nM}$ & BTZ concentration, $\mu \mathrm{M}$ \\
\hline $\begin{array}{l}\text { PEGAuNPs } \\
\text { PEGAuNPs-BT7 }\end{array}$ & $\begin{array}{l}39 \pm 1 \\
40+1\end{array}$ & $\begin{array}{l}0.6 \\
0.4\end{array}$ & $-39 \pm 4$ & $15.1 \pm 1.8$ & - \\
\hline
\end{tabular}

PEGAuNPs: pegylated gold nanoparticles; PEGAuNPs-BTZ: conjugated pegylated gold nanoparticles with Bortezomib.

Table 2 Half maximal inhibitory concentration $\left(\mathrm{IC}_{50}\right)$ and effect of BTZ alone and PEGAuNPs-BTZ on the growth inhibition $\left(\mathrm{GI}_{50}\right)$ on the pancreatic cell lines $\mathrm{S} 2$ 013, hTERT-HPNE and A549.

PEGAuNPs-BTZ: Conjugated pegylated gold nanoparticles with Bortezomib

BTZ: Bortezomib

\begin{tabular}{|c|c|c|c|c|c|c|}
\hline & \multicolumn{2}{|c|}{ S2-013 } & \multicolumn{2}{|c|}{ TERT-HPNE } & \multicolumn{2}{|c|}{ A549 } \\
\hline & PEGAuNPs-BTZ & BTZ & PEGAuNPs-BTZ & BTZ & PEGAuNPs-BTZ & BTZ \\
\hline $\mathrm{IC}_{50}, \mu \mathrm{M}$ & $0.0082 \pm 0.0002$ & $0.0122 \pm 0.0010$ & $0.4299 \pm 0.0384$ & $0.0068 \pm 0.0003$ & $0.0066 \pm 0.0005$ & $0.0281 \pm 0.0005$ \\
\hline $\mathrm{Gl}_{50}, \mu \mathrm{M}$ & $0.0023 \pm 0.0007$ & $0.0011 \pm 0.0001$ & $0.0535 \pm 0.0020$ & $0.0313 \pm 0.0051$ & $0.0054 \pm 0.0006$ & $0.0213 \pm 0.0005$ \\
\hline
\end{tabular}

PEGAuNPs-BTZ: conjugated pegylated gold nanoparticles with Bortezomib; BTZ: bortezomib. 